\title{
Aplicação do Procedimento Recursivo do Método do Elemento de Contorno para recálculo das derivadas direcionais internas em problemas escalares solucionados pelo Método dos Elementos Finitos
}

\author{
Hercules de Melo Barcelos ${ }^{1}$ \\ INMETRO, Duque de Caxias, RJ \\ Carlos Friedrich Loeffler ${ }^{2}$ \\ UFES, Vitória, ES \\ Luciano de Oliveira Castro Lara ${ }^{3}$ \\ UFES, Vitória, ES
}

\begin{abstract}
Resumo. O procedimento recursivo (MECR) é uma técnica auxiliar de elemento de contorno que foi usada com sucesso para o recálculo de derivadas potenciais e direcionais com mais precisão. Este procedimento é baseado na ideia matemática de que a equação integral de contorno é uma sentença de resíduos ponderados e sua reutilização implica uma nova minimização de erros numéricos. Neste trabalho os valores de contorno nodais calculados pelo Método dos Elementos Finitos (MEF) são reaproveitados aplicando-os na equação integral do Método dos Elementos de Contorno, visando principalmente calcular as derivadas direcionais internas do potencial. Para confirmar a robustez do modelo proposto, são realizados testes computacionais, nos quais os resultados da forma padrão do MEF são comparados com os obtidos pelo procedimento recursivo. Não apenas as derivadas de potencial interno, mas também os valores de potencial interno foram recalculados pela MECR com o objetivo de corroborar a consistência do procedimento proposto. Para avaliar a qualidade dos resultados, o exemplo apresentado contém a solução analítica para a verificação do erro médio relativo.
\end{abstract}

Palavras-chave. Método dos Elementos de Contorno, Esquema Recursivo, Método dos Elementos Finitos, Funções de Base Radial.

\section{Introdução}

Há um esforço significativo para se obter métodos numéricos mais eficientes em engenharia. Para atingir esse objetivo, algumas técnicas numéricas tradicionais foram submetidas a adaptações para obter melhor desempenho, utilizando os avanços da modelagem matemática, a velocidade de processamento do computador e sua maior capacidade de armazenamento de dados. No entanto, tais métodos numéricos também podem ser aprimorados, por exemplo, através do uso de Funções de Base Radial (FBR) [1] [4] [5] [9] [10] [14]. Será utilizado neste trabalho a FBR do tipo exponencial e a função polinomial de ordem 1 para o cálculo das derivadas internas durante a aplicação do MEF [2] [8]. Ao mesmo tempo, uma nova proposta para os cálculos dos potenciais e derivadas parciais

\footnotetext{
1 engercules@gmail.com

2loefflercarlos2@gmail.com

3 castrolara@hotmail.com
} 
internas a um domínio estabelecido é apresentada através do já conhecido MECR, um método recursivo baseado no Método dos elementos de Contorno (MEC) já apresentado por [6] [11] [13]. Neste caso, os valores de contorno obtidos pelo FEM serão utilizados, onde os potenciais foram obtidos de forma direta e as derivadas de contorno através dos valores calculados no baricentro dos elementos com lado tangente ao contorno. Para os valores de canto foi realizado uma aproximação peloo Método das Diferenças Finitas (MDF) atrasada.

O exemplo numérico que será estudado neste artigo foi extraído da dissertação de [12], sendo um problema de Laplace (vide [3] e [7]) desenvolvido no sistema de coordenadas polares, o qual apresenta as condições de contorno de Dirichlet e Neumann no domínio $\Omega(\mathrm{X})$ definido. O comportamento da curva de erros percentuais médios relativos (ARE\%) é avaliado em função do refinamento da malha para o potencial interno e a derivada potencial. O exemplo possui a solução analítica para servir de parâmetro para avaliação da precisão.

\section{Equação Integral do MECR}

O modelo apresentado trata-se de um processo estacionário em meio homogêneo, regido pela Equação de Laplace, dada pela Equação (1). Em notação indicial:

$$
u,{ }_{i i}(X)=0
$$

A equação clássica do MEC em sua forma integral inversa pode ser encontrada na literatura [4] e escrita conforme a Equação (2):

$$
c(\xi) u(\xi)+\int_{\Gamma} u(X)\left[q^{*}(\xi ; X)\right] d \Gamma(X)-\int_{\Gamma} q(X)\left[u^{*}(\xi ; X)\right] d \Gamma(X)=0
$$

Para valores internos, o termo $c(\xi)$ é igual a 1 [10] e desta forma os valores de potencial interno podem ser obtidos através da Equação (3)

$$
u(\xi)_{\text {int }}=-\int_{\Gamma} u(X)\left[q^{*}(\xi ; X)\right] d \Gamma(X)+\int_{\Gamma} q(X)\left[u^{*}(\xi ; X)\right] d \Gamma(X)=0
$$

A Equação (3) é utilizada durante a aplicação do MECR para o cálculo dos potenciais internos do MEF, para isto, são utilizados os valores de $\mathrm{U}(\mathrm{X})$ e $\mathrm{Q}(\mathrm{X})$ de contorno prescritos encontrados pelo MEF, esta ação pondera os resíduos aumentando a precisão dos valores de $u(\xi)_{\text {int }}$. Ao derivar a Equação (3) em relação a $x_{1}$ e a $x_{2}$ obtêm-se as equações integrais de contorno para o cálculo das derivadas parciais internas $d u(X)_{i n t} / d x_{i}$ ao domínio $\Omega(X)$.

Com relação ao MEF, o cálculo das derivadas internas é feito derivando a função clássica polinomial de ordem 1 dentro de cada elemento, calculando assim os fluxos em seu baricentro. o mesmo procedimento é feito utilizando a $\mathrm{FBR}$ exponencial $e^{\alpha r}$ onde $\mathrm{r}$ é a distância euclidiana do baricentro do elemento com relação aos vértices. É importante destacar que o elemento finito utilizado é do tipo triangular

\section{$3 \quad$ Experimento numérico}

O modelo solucionado pode ser observado pela Figura 1 e corresponde ao corte de uma chapa em forma de L com simetria em AD apresentada por [12].

A solução analítica para este problema é dada em coordenadas polares conforme a equação (4)

$$
u(r, \Theta)=r^{2 / 3} \operatorname{sen}(2 \Theta / 3)
$$




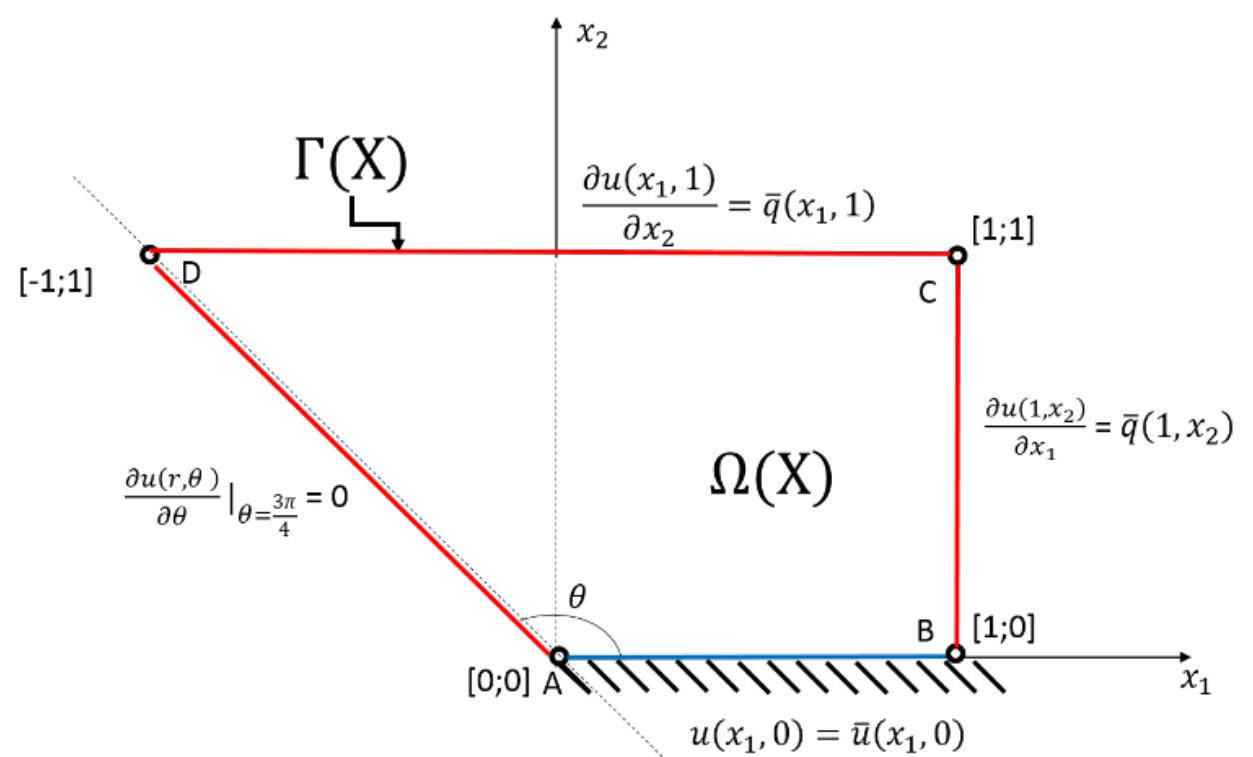

Figura 1: Região definida pelo corte $\mathrm{AD}$ para o experimento.

Com a aplicação do gradiente de $\mathrm{u}(\mathrm{r}, \Theta)$, foi encontrado o valor analítico para as derivadas direcionais conforme equação (5)

$$
\vec{\nabla} u(r, \Theta)=(2 / 3) r^{-1 / 3} \operatorname{sen}(2 \Theta / 3) e_{r}+(2 / 3) r^{2 / 3} \cos (2 \Theta / 3) e_{\Theta}
$$

A Tabela 1 representa a discretização da malha utilizada para a aplicação do MEF.

Tabela 1: Característica das malhas utilizadas no MEF

\begin{tabular}{ccccc}
\hline Nomeclatura & $\begin{array}{c}\mathrm{N}^{\mathbf{0}} \text { total } \\
\text { de pontos }\end{array}$ & $\begin{array}{c}\mathrm{N}^{\mathbf{O}} \text { total de } \\
\text { pontos no contorno }\end{array}$ & $\begin{array}{c}\mathrm{N}^{\mathbf{O}} \text { total de } \\
\text { elementos finitos }\end{array}$ & $\begin{array}{c}\mathrm{N}^{\mathbf{O}} \text { total de } \\
\text { pontos internos no } \\
\text { baricentro dos } \\
\text { elementos finitos }\end{array}$ \\
\hline Malha 1 & 19 & 12 & 24 & 22 \\
Malha 2 & 61 & 21 & 96 & 92 \\
Malha 3 & 217 & 48 & 381 & 376 \\
Malha 4 & 817 & 96 & 1536 & 1520 \\
Malha 5 & 3169 & 192 & 6144 & 6112 \\
\hline
\end{tabular}

Na Figura 2, o MECR ( ou RBE) obteve bons resultados para os valores de potencial interno, superando os métodos tradicionais MEF (ou FEM) e MEC (ou BEM), porém a perda de monotonicidade observada nas Figuras 3 e 4 se deve ao fato da descontinuidade presente no problema, além do mau condicionamento da malha para o cálculo dos fluxos internos. Como visto na Malha 5, esse problema pode ser resolvido por meio do refinamento da malha ou pela realização de testes em malhas não estruturadas. Observa-se também que para malhas com menor refinamento, os valores de RBE com contorno FEM apresentaram erros próximos ao melhor valor alcançado pelo MEC. 


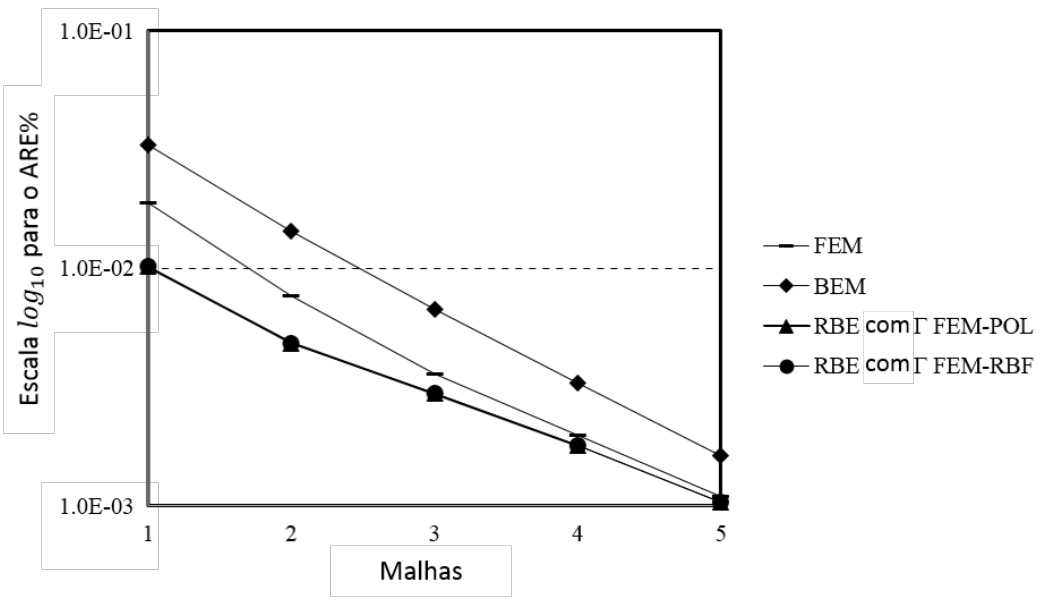

Figura 2: ARE \% para o potencial interno.

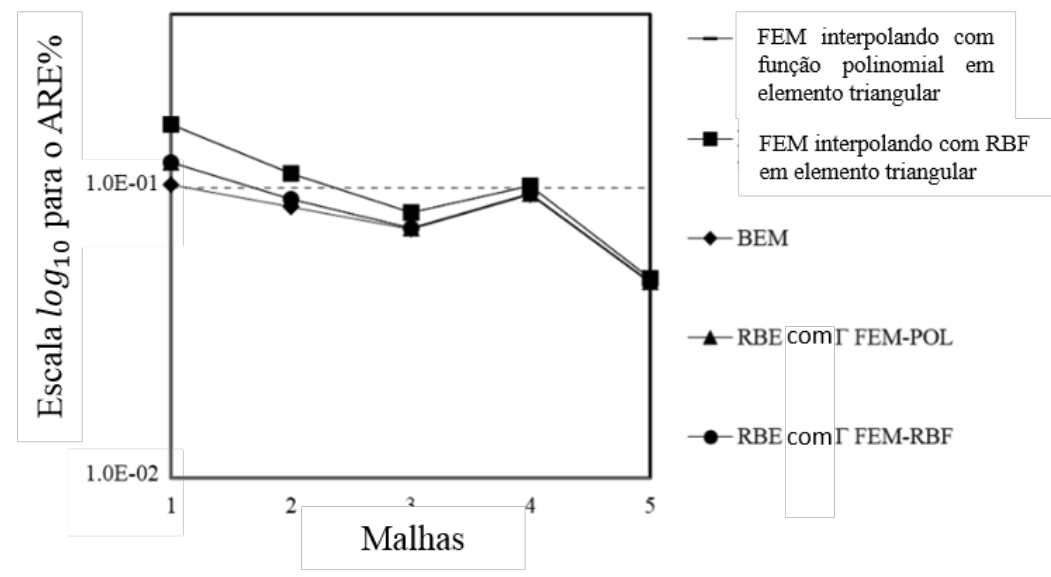

Figura 3: ARE \% para a derivada direcional $d u / d x_{1}$. 


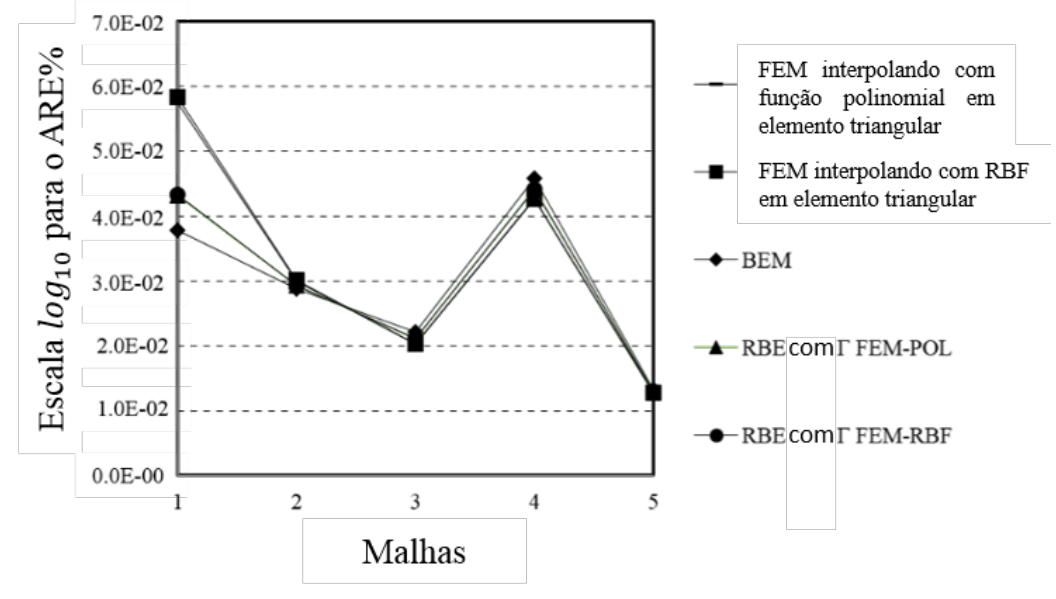

Figura 4: ARE \% para a derivada direcional $d u / d x_{2}$.

\section{Conclusões}

O objetivo deste trabalho é mostrar que a ideia contida no procedimento do MECR pode ser aplicada com vantagem em um acoplamento com o Método dos Elementos Finitos. Os resultados comprovaram que esta associação foi efetivamente positiva, sendo bastante eficiente para melhorar a precisão dos resultados das variáveis internas e também muito simples de implementar. O exemplo resolvido apresentou boa precisão no cálculo das derivadas direcionais e também melhorou significativamente a qualidade dos valores potenciais.

O MECR introduz os valores nodais de contorno obtidos com o MEF nas equações integrais do MEC para o cálculo das quantidades internas de interesse sem a necessidade de resolver um sistema de equações, pois o objetivo é calcular os coeficientes de influência com base na integração da solução fundamental e sua derivada normal. A solução para cada ponto interno escolhido arbitrariamente é gerada separadamente com baixo esforço computacional.

Uma grande vantagem desse acoplamento se deve à velocidade computacional que os modelos discretos resolvidos pelo MEC apresentam quando grandes problemas são resolvidos. Com o acoplamento MEF - MEC, não seria necessário resolver um sistema com tantos graus de liberdade se os valores internos fossem de grande interesse; assim, o custo de obtenção de valores internos pelo MEC é muito baixo e compensador. Para trabalhos futuros objetiva-se aplicar o MECR no problema de Poisson.

\section{Agradecimentos}

Aos autores agradecem ao Inmetro e à Ufes pelo apoio insentivo a pesquisa.

\section{Referências}

[1] Barcelos, H. M. and Loeffler, C. F. The direct interpolation boundary element method applied to smoothly inhomogeneous Laplace's problems, Engineering Analysis with Boundary Elements, 2019. DOI: 10.1016/j.enganabound.2019.04.014 
[2] Bathe, K. J. Finite Element Procedures, 2st edition. Prentice Hall, United States of America, 1996.

[3] Braga, C. L. R. Physical-Mathematics Notes (in portuguese). Editora Livraria da Física, São Paulo, 2006.

[4] Brebbia, C. A., Dominguez J. Boundary elements - an introductory course, 2nd edition. WIT Press, United Kingdom, 1994.

[5] Buhmann, M. D. Basis Function: Theory and Implementations, 1st edition. Cambridge University Press, United Kingdom, 2003.

[6] Freitas, A. B., Loeffler, C. F. Performance Evaluation of the Boundary Element Recursive Procedure in Elastic Problems, J. of Eng. Math., 2015. DOI:10.1007/s10665-015-9807-9

[7] Ingber, M.S., Rudolphi, T. J. Solution of potential problems using combinations of the regular and derivative boundary integral equations, Appl. Math. Modelling, 1990

[8] Kythe, P. K. Wei, D. An Introduction to Linear and Nonlinear Finite Element Analysis, 1st edition. Springer, United States of America, 2004.

[9] Loeffler, C. F., Cruz, A. L. and Bulcão A. Direct use of radial basis interpolation functions for modelling source terms with the boundary element method, Engineering Analysis with Boundary Elements, 2015. DOI: 10.1016/j.enganabound.2014.07.007

[10] Loeffler, C. F., Mansur, W. J. A Regularization scheme applied to the direct interpolation boundary element technique with radial basis functions for solving eigenvalue problem, Engineering Analysis with Boundary Elements, 2017. DOI: 10.1016/j.enganabound.2016.10.008

[11] Loeffler, C. F. A Recursive Application of the Integral Equation in the Boundary Element Method, Engineering Analysis with Boundary Elements, 2011. DOI: 10.1016/j.enganabound.2010.05.012

[12] Prado, A. A. Uma Formulação Hipersingular do Método dos Elemento de Contorno para problemas bidimensionais, Dissertação de Mestrado, UFRJ, 1991.

[13] Ramos, V. E. S., Loeffler, C. F. and Mansur, W. J. Recursive Procedure of Boundary Element Method Applied to Poisson's Problems, Engineering Analysis with Boundary Elements, 2017. DOI:10.1016/j.enganabound.2017.06.003

[14] Wang, J. G. and Liu, G. R. A Point Interpolation Meshless Method based on Radial Basis Functions, Inter. J. for Num. Methods in Eng., 2002. DOI: 10.1002/nme.489 